AperTO - Archivio Istituzionale Open Access dell'Università di Torino

\title{
Management of infection in systemic lupus erythematosus.
}

\section{This is the author's manuscript}

Original Citation:

Availability:

This version is available http://hdl.handle.net/2318/142599

since 2016-11-02T16:05:15Z

Published version:

DOI:10.1016/j.berh.2013.07.002

Terms of use:

Open Access

Anyone can freely access the full text of works made available as "Open Access". Works made available under a Creative Commons license can be used according to the terms and conditions of said license. Use of all other works requires consent of the right holder (author or publisher) if not exempted from copyright protection by the applicable law. 
This Accepted Author Manuscript (AAM) is copyrighted and published by Elsevier. It is posted here by agreement between Elsevier and the University of Turin. Changes resulting from the publishing process - such as editing, corrections, structural formatting, and other quality control mechanisms - may not be reflected in this version of the text. The definitive version of the text was subsequently published in BAILLIERE'S BEST PRACTICE \& RESEARCH: CLINICAL RHEUMATOLOGY, 27, 2013, 10.1016/j.berh.2013.07.002.

You may download, copy and otherwise use the AAM for non-commercial purposes provided that your license is limited by the following restrictions:

(1) You may use this AAM for non-commercial purposes only under the terms of the CC-BY-NC-ND license.

(2) The integrity of the work and identification of the author, copyright owner, and publisher must be preserved in any copy.

(3) You must attribute this AAM in the following format: Creative Commons BY-NC-ND license (http://creativecommons.org/licenses/by-nc-nd/4.0/deed.en), 10.1016/j.berh.2013.07.002

The publisher's version is available at:

http://linkinghub.elsevier.com/retrieve/pii/S1521694213000545

When citing, please refer to the published version.

Link to this full text:

http://hdl.handle.net/2318/142599 


\title{
Management of infection in systemic lupus erythematosus.
}

\author{
Sciascia $\mathrm{S}^{1}$, Cuadrado MJ, Karim MY. \\ ${ }^{1}$ Lupus Research Unit, The Rayne Institute, St Thomas' Hospital, Westminster Bridge Road, London SE1 7EH, UK.
}

\begin{abstract}
Systemic lupus erythematosus (SLE) is a systemic autoimmune disease characterised by abnormal autoantibody production and clearance. This immunological background has been suggested to play a role in the susceptibility of SLE patients to infection. Moreover, drugs (most of them immunosuppressive or immunomodulating agents) used in the treatment of moderate and severe lupus give rise to a tendency for infections, including opportunistic ones. Infections may mimic the exacerbations of SLE, leading to confusion over the diagnosis and appropriate treatment. Despite increased awareness of this problem, infections remain a major source of morbidity and mortality in SLE. There are various strategies which can be applied to try and reduce the risk of infection in SLE patients. Options include vaccinations, antibiotic/antiviral prophylaxis and intravenous immunoglobulins.
\end{abstract}

Keywords

Bacterial viral fungal flare; Vaccination; Vaccine antibiotic prophylaxis; Immunoglobulin

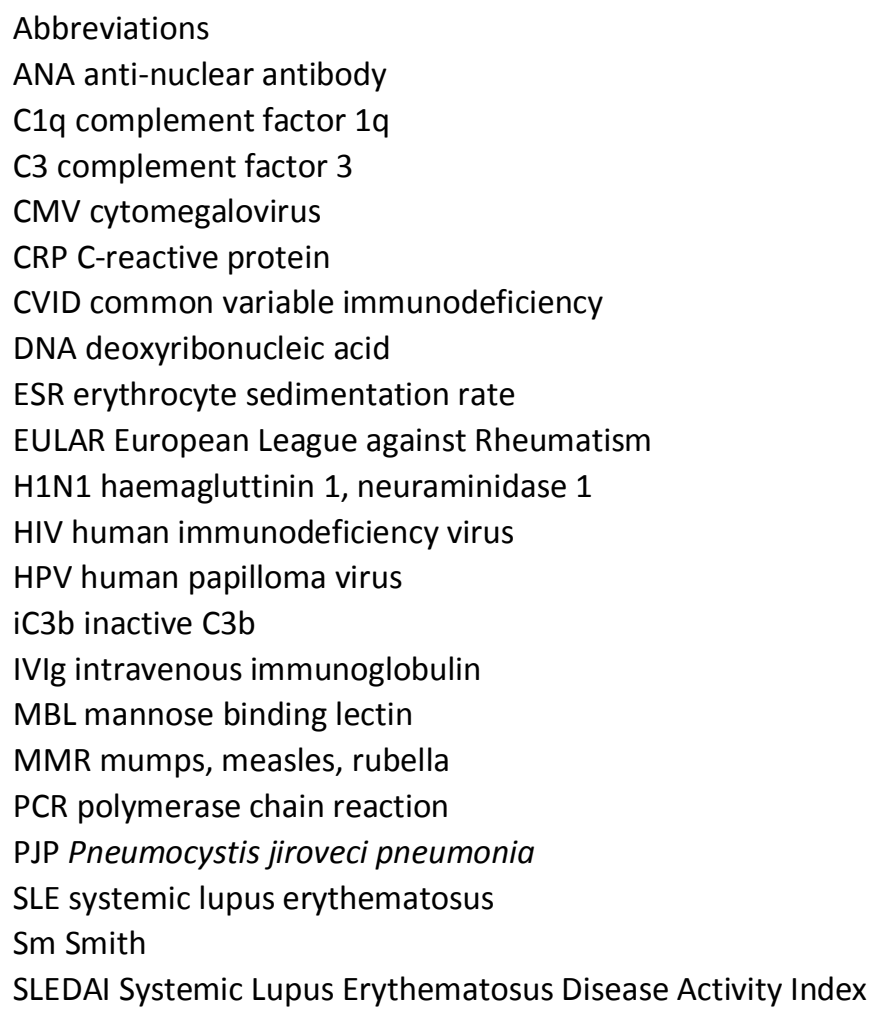

Introduction

Systemic lupus erythematosus (SLE) is a systemic autoimmune disease characterised by abnormal autoantibody production and clearance. This immunological background has been suggested to play a role in the susceptibility of SLE patients to infections [1]. Moreover, drugs (most of them immunosuppressive or immunomodulating agents) used in the treatment of moderate and severe lupus give rise to a tendency for infections, including opportunistic ones. 
Infections may mimic the exacerbations of SLE, leading to confusion over the diagnosis and appropriate treatment. Despite increased awareness of this problem, infections remain a major source of morbidity and mortality in SLE. Infectious diseases are still the most frequent causes of death in SLE in the first year after onset [2]. The increase in SLE deaths associated with infection, especially pneumonia and septicaemia, is worrisome, especially in some areas of the globe [3]. Indeed, survival rates for SLE patients in developing countries are comparatively lower than those reported in industrialised countries, with early death from infection and active disease [4].

In this review, we aim to review infections in SLE and appropriate prevention strategies.

\section{Characteristics of infection in SLE}

Infections are known to be a major cause of morbidity and mortality in SLE [1]. Several studies evaluated the characteristics of major infections in SLE patients requiring hospitalisation [5], * [6] and [7]. According to these studies, infections that SLE patients developed were attributed to the same pathogens as in the general population and included community-acquired pneumonia, urinary tract infection and vaginal infection, and some patients may develop tuberculosis. However, despite the pathogens often being the same as in the general population, the clinical manifestations of the infections can be atypical, due to an abnormal immunological response or due to ongoing treatment. Careful inspection and monitoring and timely collection of the specimens for bacterial culture are warranted to avoid misdiagnosis.

Some patients also develop viral, fungal and protozoan infections. Rarely, multiple organisms can be found [8]. Usually, in an outpatient setting, infections are non-life-threatening ones. Zonana-Nacach et al. reported that in this scenario, infections are associated with disease activity only, independently of socio-demographic and therapeutic factors [5]. However, it is noteworthy that infection in SLE can require hospitalisation, especially when concomitant with a flare (mainly involving the kidney or central nervous system) or when therapy with steroids or cyclophosphamide is ongoing [1].

\section{Predictors of major infections in SLE}

Several studies have analysed the prevalence and associated clinical and laboratory features of infection in SLE *[8], [9], [10] and [11]. Common themes are medications, including use of steroids and/or cyclophosphamide or high-disease activity as measured by the SLE Disease Activity Index [12].

Recently, Ruiz-Irastorza and co-workers [13] in a nested case-control study design used within the prospective Lupus-Cruces cohort analysing 249 patients found that the risk of major infections in patients with SLE is mostly influenced by treatment. Prednisone treatment, even at moderate doses, increases the risk, whilst antimalarials have a protective effect [13]. In detail, it was shown that the prednisone dose at the time of the event had a facilitating effect on infections, in agreement with previous studies [9], [10] and [11]. Moreover, it is worthy of note that the median dose of patients with major infections was only $7.5 \mathrm{mg}$ day-1, with an 11-times higher risk of suffering a serious infection for each increase of $10-\mathrm{mg}$ day-1 prednisone.

\section{SLE, infections and mortality}

When the frequency, characteristics of the main causes and prognostic significance for morbidity and mortality in 1000 patients with SLE during a 5-year period were analysed, the most frequent causes of death were active SLE, infections and thromboses [14]. In the long-term follow-up of the same study, infection was the present in $36 \%$ of the 1000 patients and most frequent causes of death were confirmed to be similarly divided among active SLE (26.5\%), thromboses (26.5\%) and infections (25\%). A survival probability of $92 \%$ at 10 years was found [15].

In a retrospective study performed to describe the characteristics associated with a poor outcome in SLE patients admitted to hospital during a 1-year period, infection was found to be the second cause of hospitalisation after clinical flare of SLE [16]. 


\section{Infections according to nature of microbe Bacterial infections}

A wide variety of infectious pathogens have been recognised in SLE. The most frequent types of infections are respiratory, urinary tract and soft-tissue infections [12]. Most infections are caused by common pathogens and include Staphylococcus aureus, Streptococcus pneumoniae, Escherichia coli and Pseudomonas aeruginosa. Indeed, common pathogens that often behave more aggressively than in the healthy population. ${ }^{*}[12]$ and ${ }^{*}[17]$ An increased incidence of Salmonella infection and pneumococcal sepsis is also observed [18].

\section{S. pneumoniae}

S. pneumoniae is a Gram-positive bacterium, which is a common cause of community-acquired pneumonia, meningitis and septicaemia. Several studies indicate that patients with SLE have an increased frequency and severity of $S$. pneumoniae infections, accounting for $6-18 \%$ of all bacterial infections in these patients *[17] and [20]. It has been suggested that some defective mechanisms in SLE patients can underpin this increased susceptibility to S. pneumoniae infections. Recently, Goldblatt et al. [20] reported that opsonisation of S. pneumoniae with complement factor $3 \mathrm{~b} /$ inactive $\mathrm{C} 3 \mathrm{~b}(\mathrm{C} 3 \mathrm{~b} / \mathrm{iC} 3 \mathrm{~b})$ was significantly reduced in serum from patients with SLE compared with patients with non-SLE rheumatic disease and healthy controls, suggesting that a failure to appropriately activate the immune system via complement may contribute to the increased susceptibility of SLE subjects to infections and may correlate with a risk of pneumonia in a subgroup of SLE patients.

\section{Salmonella}

Infection with Salmonella species is recognised to be more common in SLE patients than the normal population and may be due to splenic dysfunction or to a defect in opsonisation as previously described [19] and [20]. The risk factors of mortality for Salmonella infection have been recently analysed in a cohort of SLE patients hospitalised in a medical centre in Taiwan [21]. Patients with Salmonella infection associated with lupus flare or re-infection with Salmonella species have been found to be the strongest factor associated with a higher risk of mortality [21]. In another study, retrospectively reviewing 50 SLE patients diagnosed with bacteriologically proven non-typhoidal salmonellosis over a 20 -year period, it was found that most episodes were bacteraemic without a localising focus, and some patients were afebrile. Mortality in general occurred from concomitant septic shock and major organ failure from active lupus (mainly renal) [22]. It is noteworthy that osteomyelitis of the long bones due to salmonella can occur in SLE patients [23]. Active SLE or co-existent underlying systemic disease, chronic renal failure, and immunosuppressive agents were shown as main predisposing factors [23]. Recently, Navarra et al. [24] described the spectrum of Salmonella infections among Filipino patients with SLE, with typhoid fever and septic arthritis as the most common presentation. Atypical involvement included softtissue abscess and meningitis, with the worst prognosis noted in those with sepsis syndrome.

\section{Klebsiella}

Some studies showed that Klebsiella was one of the leading causes of Gram-negative bacteraemia in the general population [25]. SLE patients infected with Klebsiella were found to have lower probabilities of 14-day survival [26] in a study analysing the short-term survival of patients with SLE after bacteraemia episodes.

It is noteworthy that sera from patients with Klebsiella pneumoniae were found to contain high titres of the common anti-DNA idiotype [27]. However, the presence of autoantibodies in the serum of patients with Klebsiella infections may be the result of non-specific stimulation due to bacterial polyclonal activation. However, there might also be a specific stimulus triggered by idiotypic cross-reaction between autoantibodies and anti-Klebsiella antibodies [28].

\section{Mycobacterium tuberculosis}


The reported prevalence of $M$. tuberculosis infection in SLE patients ranges widely from $5 \%$ to $30 \%$ [29] and [30]. The higher prevalence of tuberculous infections in SLE is attributed to multiple immune abnormalities observed in these patients and to the immunosuppressant therapy [31]. The clinical presentation of M. tuberculosis infection seems to be different in SLE patients when compared with the general population; in fact, more frequent extrapulmonary involvement as well as more extensive pulmonary involvement and a high relapse rate even if treated with prophylactic isoniazid have been reported [30] and [32]. Mycobacterial infection and SLE may have a similar presentation and may mimic each other. In an individual patient, the differential diagnosis is crucial. In a retrospective analysis involving more than 3000 SLE patients, Hou et al. [33] documented 19 lupus patients with 21 episodes, 10 of which were pulmonary while the other 11 episodes were extrapulmonary (joint, cutaneous or visceral-organ involvement). Fever and cough were found to be the most common manifestations of tuberculosis. However, Sayarlioglu et al. [34], comparing lupus patients without and with tuberculosis, found that arthritis and renal disease were significantly higher in the latter group, underling the importance of an accurate diagnostic approach.

\section{Opportunistic infections}

Increasing evidence indicates that opportunistic infections contribute to the infectious mortality in SLE, as stated before. The burden of opportunistic infections in SLE is complex; often, they are under-reported due to difficulties in diagnosis, as they can mimic or be superimposed upon active lupus. Listeriosis, nocardiosis, candidiasis, cryptococcal meningitis, Pneumocystis jiroveci pneumonia (PJP) and invasive aspergillosis are described in patients with SLE [1]. Sometimes, even more rare infections are reported to occur in SLE, such as haemotrophic mycoplasma [35].

Cases of SLE with fungaemia or invasive fungal infection are rare but life-threatening conditions in SLE [36]. Severe Candida infection is the most frequently identified opportunistic fungal infection in several SLE series, associated with steroid and cytotoxic drug therapy [37]. Nocardial infections have been also described in steroid-treated SLE patients, and pneumonia and brain abscess are the most frequent clinical presentations [38] and [39]. Taken together, all the studies stressed that active lupus disease (SLEDAI > 7) is probably the main risk factor for opportunistic infection. It is noteworthy to remember that low prednisolone doses before fungal infection or high prednisolone doses following fungal infection are associated with higher mortality [37].

\section{Viral infections}

The most commonly reported viral infections in patients who have SLE are parvovirus B19 (HPV-B19) (there are more than 30 reports of primary B19 infection reported as lupus-like syndrome) [37] and cytomegalovirus (CMV) (predominantly presenting in severely immunosuppressed patients). It is not among the purposes of this review to analysis the causative role of virus in the pathogenesis of SLE. Herewith, we focus on the clinical settings when the two conditions, SLE and viral infection, co-exist.

Ramos-Casals et al. [40] described the largest series of acute viral infections in SLE patients. Among 25 patients diagnosed with new-onset SLE, HPV-B-19, CMV, Epstein-Barr virus and hepatitis A were concomitantly detected. In patients already diagnosed with SLE, symptoms related to infection mimicked a lupus flare due to disseminated viral infection and a severe, multi-organ process similar to that described in catastrophic antiphospholipid syndrome was reported. Mortality was high, with 12 patients dying due to infection.

\section{Parvovirus B19}

The occurrence of HPV-B19 infection has been documented in patients with SLE, in particular in relation to disease onset. The main reported clinical manifestations were fever, articular involvement, cutaneous lesions, lymphadenopathy, hepato and/or splenomegaly, serositis, renal involvement and cerebral impairment. Cytopaenia was also frequently observed. Thus, the differential diagnosis between HPV-B19 infection and SLE flare is a real challenge, also because HPV-B19 infection may induce a serological profile mimicking a flare. Elevated titres of double-stranded DNA, Sm (Smith), nuclear ribonucleoprotein, Ro-SSA, La-SSB, cardiolipin and/or beta2-glycoprotein I antibodies were reported in concomitant to B19 infection [41]. The B19 infection 
has usually a self-limiting course; nevertheless, in immunocompromised SLE patients symptoms may persist several months after the viral infection and induce severe clinical settings.

\section{CMV}

Sekigawa et al. [42], reporting SLE patients with spatially related SLE and CMV, emphasised the main features of the complex relationship between SLE and viral infection: a) CMV infection and SLE exacerbation may be impossible or difficult to distinguish, $b$ ) the development of SLE may be triggered by a CMV infection and 3) existing SLE may undergo an exacerbation following a CMV infection. Notably, CMV infection can be considered as an opportunistic infection, when affecting SLE patients on chronic steroids and/or immunosuppressive agents. Among the possible CMV-related manifestations, retinitis has to be kept in mind, especially when ongoing therapy includes both azathioprine and low-dose corticosteroid [43].

\section{HIV}

Interestingly, SLE has a lower incidence in the human immunodeficiency virus (HIV)-infected population when compared to the general population [44]. It has also been suggested that SLE may be influenced by HIV infection. It has been suggested that the immunosuppression resulting from HIV infection can prevent the emergence of SLE, as the immunosuppressive effect of HIV may inhibit the development of autoimmune diathesis [45]. To date, several cases of concomitant association between the two diseases have been reported, but the diagnosis was simultaneous in very few of those. Very recently, Carugati et al., reporting two cases and review of the literature, concluded that SLE could occur despite the loss of immunocompetence caused by HIV infection. Moreover, they stated that SLE and HIV infection might influence each other possibly through immunologic mechanisms determining awkward manifestations [46].

\section{Serological markers of infection in SLE}

Conventional biomarkers of lupus flares are hypocomplementaemia, anti-double-stranded-DNA antibodies and erythrocyte sedimentation rate. Their value to predict disease flares and efficacy of therapeutics has been proven; however, even taken together, they are not specific and lack diagnostic accuracy in differentiating between flares and infections [47]. In SLE, unlike in other rheumatic diseases where increase in levels has been observed, changes in C-reactive protein (CRP) level have been less frequently observed during disease flares, proposing this marker as a valid tool to discriminate infection and lupus activity [48]. Albeit with some limitations (such as the presence of arthritis and serositis), to date CRP levels and erythrocyte sedimentation rate/C-reactive protein (ESR/CRP) ratio seem to be the best marker to differentiate SLE activity from infection. Other biomarkers such as procalcitonin, and autoantibodies against complement fraction C1q, have been investigated to distinguish infections from other inflammatory processes but further studies are warranted.

\section{Prevention of infection in SLE}

As illustrated in the first part of this article, predisposition to infection with microbial agents is a major problem in SLE. If patients develop either atypical or recurrent infections, then this is a challenge to the clinician. There is a range of predisposing factors which may be present in SLE patients (see Table 1). There are various strategies which can be applied to try to reduce the risk of infection in SLE patients. Options include vaccinations, antibiotic/antiviral prophylaxis and intravenous immunoglobulins (IVIg).

\section{Vaccinations}

Patients with SLE are at an increased risk of infection, especially if they are taking immunosuppressive medication. Vaccination can be considered as a possible strategy. The history of vaccination in SLE patients has been controversial. This is because in certain cases, vaccination can trigger a flare of the disease. However, a number of reviews have recently been undertaken and seem to be in favour of vaccination, that is, the risk benefit is for vaccination to reduce infection risk compared with the risk of flare [49]. In their own practice, the authors opt for vaccination unless there is a previous history of vaccination-related disease flare. If possible, 
vaccination should be administered before commencing immunosuppressive medication, as once this is started then live vaccines are contraindicated, and responses to non-live vaccines may be suboptimal. Non-live (inactivated/killed or subunit) vaccines can be used in patients on immunosuppressant therapy [49]. It is also best to vaccinate, if possible, when the lupus is quiescent [50].

The major questions with respect to vaccination in SLE and other autoimmune rheumatic diseases are as follows:

1. Are they safe?

2. Do they trigger disease flares?

3. Do they work?

4. Which vaccines are important?

\section{Are they safe?}

Killed or subunit vaccines are safe to administer to SLE patients on immunosuppression. However, live vaccines are contraindicated in patients on immunosuppressive medications according to expert opinion from European League against Rheumatism (EULAR). Expert opinion from EULAR also suggests that vaccines should be administered while disease activity is quiescent [50]. Abu-Shakra also suggested avoiding vaccines when lupus is very active [51].

\section{Do they trigger disease flares?}

Vaccines may trigger the generation of autoantibodies, which are usually of short term and of little clinical significance. Hence, more commonly, induction of autoantibodies but not autoimmune disease may occur. For example, induction of cardiolipin antibodies, but not $\beta 2$-glycoprotein-I antibodies, was seen in SLE patients and healthy controls [52]. However, in individual cases vaccines may cause SLE flares. No specific clinical or laboratory variables have been shown to be predictive of flare of systemic lupus erythematosus following vaccination [51].

\section{Do they work?}

Vaccinations, if possible, could be administered before commencing immunosuppressive medication as vaccination responses may be blunted in patients receiving high-dose corticosteroids or immunosuppressive medication. If possible, the authors suggest vaccination before starting immunosuppressive therapy or biologics. For example, rituximab, as a B-cell-depleting medication, has a significant effect on vaccine responses. It is best to administer vaccines either 4 weeks before or 6 months after rituximab treatment [50].

O'Neill and Isenberg (2006) commented that the majority of SLE patients have an appropriate response to vaccination, but a significant minority do not [49]. Response to hepatitis-B vaccination may be impaired and serological responses should be assessed post vaccination. They speculated that disease activity or immunosuppressive drugs were risk factors of poor response, rather than intrinsic abnormalities of immune function in SLE.

Response to influenza vaccination was lower in those SLE patients with haematologic criteria, or on prednisolone or of European American ancestry. After vaccination, low respondents were more likely to have disease flares and to have increased titres of anti-nuclear antibodies (ANAs) [53]. Azathioprine impaired response after influenza vaccination, though most patients still developed protective responses [54] and [55].

\section{Which vaccines are important?}

\section{Pneumococcus}

Respiratory infections are common in patients with SLE. Vaccination against the Pneumococcus bacterium should be with Pneumovax 23, the 23-valent polysaccharide vaccine, as the first line, to give the broadest coverage. If the patient does not respond to Pneumovax 23, then Prevenar 13, the 13-valent conjugated 
vaccine, can be considered. Where possible, it is important to ensure that patients are responding to vaccinations by checking pre- and post-vaccination antibody levels. This is routinely possible for Pneumococcus, and serology is also available for other organisms such as Haemophilus influenzae, Meningococcus, diphtheria and tetanus toxoids and mumps, measles and rubella viruses.

The duration of protective antibody status may be shorter in patients on immunosuppressive therapy. Hence, the standard advice of a 5-year interval for pneumococcal vaccination may not hold true. Measurement of antibody levels, namely serotype-specific pneumococcal antibodies, may help guide vaccination frequency. Elkayam et al. showed that most patients did respond to the pneumococcal vaccine, but five $(20.8 \%)$ of 24 patients with SLE responded to either none or only one of the seven serotypes tested [56].

\section{Tuberculosis}

Bacillus Calmette-Guérin (BCG) is not recommended because most cases are related to reactivation, not de novo infection; BCG is not always protective against tuberculosis in adults; there is a risk of BCGosis in patients on immunosuppressive therapy. In Western countries, there is not a need for anti-tuberculosis prophylaxis. However, in areas where tuberculosis is common, for example, India, or in patients of certain ethnic origins, there may be a case for anti-tuberculosis prophylaxis [57].

\section{Influenza}

One should consider vaccination with seasonal influenza annually and with the H1N1 influenza A strain. Studies suggest that patients do mount an immune response but perhaps less than healthy controls. Wiesik-Szewczyk et al. (2010) showed a lower seroprotection rate after inactivated influenza vaccine in SLE patients compared with healthy controls [58]. There were one severe and six mild-to-moderate flares observed in 62 SLE patients. Mathian et al. showed that impaired efficacy of H1N1 influenza A vaccine was associated with immunosuppressive medication or lymphopaenia [59].

Another possible good reason for antimalarials is the following: Borba et al. showed reduced response to influenza $\mathrm{A} / \mathrm{H} 1 \mathrm{~N} 1$ in patients taking $>20 \mathrm{mg}$ day-1 prednisolone, or on immunosuppressive medication or receiving both [60]. The group of patients taking chloroquine had a non-significant difference in seroconversion compared to the SLE no-therapy group. However, these findings would need to be corroborated in larger studies.

Azathioprine impaired the response after influenza vaccination in SLE but most patients managed to develop protective antibody levels [54] and [55].

\section{Human papilloma virus}

Cervical smear abnormalities are common in SLE, which may progress to cervical cancer. It may therefore be worth considering the use of the quadrivalent HPV vaccination, Gardasil [61]. Mok et al. reported the Gardasil vaccine to be safe and reasonably effective in a group of 50 SLE patients [62].

Thrombo-embolic events after Gardasil vaccination have been reported. Of the 31 reported cases, $90 \%$ had a risk factor, in fact antiphospholipid syndrome in two cases [63]. It is important to be aware of antiphospholipid antibody status in SLE patients so that appropriate measures can be taken.

\section{Herpes zoster}

There are separate varicella zoster (VZV; e.g., Varivax) and herpes zoster (e.g., Zostavax) vaccines. Licenced herpes zoster vaccine is a lyophilised preparation of a live, attenuated strain of VZV, which is the same strain used in the varicella vaccines. However, its minimum potency is at least 14-times higher than the potency of a single-antigen varicella vaccine. Although live vaccines are to be avoided in general for patients receiving immunosuppressive therapy, the Advisory Committee on Immunization Practices (ACIP) has made recommendations for the herpes zoster vaccine. [64] The committee has proposed that the dose of immunosuppressive makes a difference, for example, patients could receive the herpes zoster vaccine if taking 
$<0.4 \mathrm{mg} \mathrm{kg}-1$ week -1 methotrexate, $<3.0 \mathrm{mg} \mathrm{kg}-1$ azathioprine and a moderate dose of corticosteroids $<20 \mathrm{mg}$ day-1. Varicella vaccine has been administered without subsequent infection in HIV-infected children with a CD4 percentage $\geq 15 \%$ or a CD4 count $\geq 200 \mathrm{~mm}-3$ [65]. Varicella vaccine has also been administered to HIVinfected subjects with $C D 4 \geq 400$ cells $\mu \mathrm{l}-1$, but was only modestly immunogenic [66]. There are unpublished studies for the herpes zoster vaccine in adult HIV patients with a CD4 count $\geq 200 \mathrm{~mm}-3$ (http://www.clinicaltrials.gov/ct2/show/NCT00851786?term=zostavax+hiv\&rank=1) and in older patients on treatment with prednisone $5-20 \mathrm{mg}$ day-1

(http://www.clinicaltrials.gov/ct2/show/NCT00546819?term=zostavax+corticosteroid\&rank=1). Specialist advice should be sought from a consultant virologist, when considering live vaccinations in patients receiving immunosuppression.

\section{Measles, mumps and rubella}

Measles, mumps and rubella (MMR) is a live vaccine, and most individuals in the UK would have received the MMR vaccine as part of their standard childhood immunisation schedule. It is possible to check antibody levels to mumps, measles and rubella. Theoretically, as a live vaccine, MMR is contraindicated in patients on immunosuppressive therapy. However, the MMR vaccine has been administered without subsequent infection to paediatric patients 2 years after bone marrow transplantation [67].

EULAR points out that MMR, varicella and herpes zoster vaccine might be exceptions to this rule and may be considered in mildly immunosuppressed patients with autoimmune rheumatic disease on a case-by-case basis. The authors believe that this is a grey area, and that individual decisions should be made between the rheumatologist, clinical immunologist and virologist.

\section{Antibiotic prophylaxis}

No great deal of evidence exists as a basis for antibiotic prophylaxis, though in certain cases use of such an approach makes common sense. For example, in patients who develop recurrent infections with a particular organism, it may be worth considering prophylactic antimicrobials, at least while they remain on immunosuppressant medication. For example, a patient with recurrent herpes zoster infection could be considered for prophylaxis with valaciclovir. The herpes zoster vaccine (Zostavax) is live and hence cannot be considered in patients receiving a certain level of immunosuppression. A patient developing $\mathrm{CMV}$ retinitis on azathioprine could be considered for the following:

- checking thiopurine methyltransferase levels,

- an alternative immunosuppressant and

- prophylaxis with valganciclovir.

Gilliland and Tsokos (2002) suggested antimicrobial prophylaxis in cohorts of patients with increased prevalence of certain infections, those who receive heavy doses of immunosuppressive agents or those undergoing procedures associated with temporary bacteraemia [68].

\section{Endocarditis}

Zysset et al. (1987) recommended that patients with SLE who have valvular abnormalities should receive endocarditis prophylaxis before invasive dental or genitourinary procedures [69].

\section{Tuberculosis}

Prophylactic treatment against tuberculosis should be considered in certain ethnic groups of patients with SLE, and in certain parts of the world where tuberculosis is common [57]. It is not required in low-risk patients in the Western world. 
CMV infection is an uncommon but potentially fatal opportunistic infection in SLE. Yoon et al. suggested more active CMV vigilance and consideration of polymerase chain reaction (PCR)-based CMV prophylaxis in CMV PCRpositive patients with SLE undergoing intensive immunosuppressive therapy [70].

\section{PJP}

Gupta et al. reviewed the occurrence of PJP in SLE. They found a low frequency of PJP in SLE patients on cyclophosphamide of $0.1588 \%$ [71]. They did not recommend routine use of trimethoprim-sulphamethoxazole for PJP prophylaxis in SLE patients on cyclophosphamide, except in those with elevated risk, that is, with severe leucopenia, lymphopaenia, high-dose corticosteroids, hypocomplementaemia, active renal disease and higher mean Systemic Lupus Erythematosus Disease Activity Index (SLEDAI) score [71] and [72].

\section{Splenectomy and complement}

Patients may have had splenectomy as part of SLE management, such as for thrombocytopaenia. In these cases, vaccination with encapsulated bacteria is essential. This should include $S$. pneumoniae, H. influenzae type $b$ and Neisseria meningitidis (A, C, W135 and Y). They should also receive antibiotic prophylaxis with penicillin $\mathrm{V}$. In addition, there is evidence of splenic dysfunction in SLE [73], with defective clearance of immune complexes, which could include microbial antigens. Low complement levels and acquired reduction of complement receptor type 1 on red cell membranes reduce immune complex delivery to the spleen [74].

Hepburn and Davies (2002) stress the important relationship between hypocomplementaemia, splenic dysfunction and infection in SLE [74]. Infection with S. pneumoniae and N. meningitidis appears to be especially important. They have recommended antibiotic prophylaxis in SLE patients with chronic acquired hypocomplementaemia, as well as in the very rare genetic complement deficiencies with SLE. With respect to the latter, 13 out of 41 patients with C1q deficiency had recurrent bacterial infections, including meningitis and pneumonia [75].

\section{Intravenous immunoglobulin}

Replacement therapy with IVIg is appropriate for primary immune deficiency and can also be very helpful in patients with secondary immune deficiency. There are a number of different humoral defects which have been described in SLE patients. For example, common variable immune deficiency (CVID), drug-induced hypogammaglobulinaemia, hypogammaglobulinaemia related to nephrotic syndrome and specific antibody deficiency are all reported [76]. In some cases, the patient may be well though the immunoglobulin levels may be low. In these cases, a wait-and-see policy may be appropriate. For example, immunoglobulin levels in patients with drug-induced or nephrotic syndrome-related hypogammaglobulinaemia may improve over time. Treatment of the nephrotic syndrome should improve the hypogammaglobulinaemia. Drugs implicated in causation of hypogammaglobulinaemia include cyclophosphamide and rituximab. The latter does not tend to cause hypogammaglobulinaemia after the initial course but only after repeated cycles. Venhoff et al. recommended surveying patients post cyclophosphamide and rituximab treatment for serum immunoglobulin levels and persisting hypogammaglobulinaemia [77]. In some patients, hypogammaglobulinaemia may be associated with an increased risk of infection, particularly of the respiratory tract. Some patients may have specific antibody deficiency: that is, normal immunoglobulins but an inability to mount an antibody response to specific bacteria, even after vaccinations.

Therefore, criteria for replacement IVIg therapy in SLE patients should be the presence of hypogammaglobulinaemia or specific antibody deficiency, together with a history of recurrent infection. In specific antibody deficiency, an additional condition should be that they have not responded to treatment with antibiotic prophylaxis.

The approach to IVIg in these situations would be to administer a replacement dose, rather than the high-dose immunomodulatory approach used for suppressing autoimmunity. Immunoglobulin infusions can be administered every 3-4 weeks at a dose of approximately $0.4 \mathrm{~g}$ kg weight-1 month-1. Treatment for these patients should be supervised by a consultant clinical immunologist. 
Monitoring for IVIg consists of measurement of the trough immunoglobulin $\mathrm{G}$ (IgG) level, obtained immediately prior to an infusion. The target trough level should be $5-10 \mathrm{~g} \mathrm{I}-1$, but towards $10 \mathrm{~g} \mathrm{I}-1$ if there is established respiratory disease such as bronchiectasis. Additional monitoring has traditionally been full blood count (FBC), liver function tests (LFTs), hepatitis B surface antigen and hepatitis-C PCR. However, there have been no cases of IVIg-acquired viral hepatitis since the mid-1990s, and hence the hepatitis monitoring may become superfluous.

\section{References}

[1] Zandman-Goddard G, Shoenfeld Y. Infections and SLE. Autoimmunity 2005;38:473-85.

[2] Mok CC, Kwok CL, Ho LY, Chan PT, Yip SF. Life expectancy, standardized mortality ratios, and causes of death in six rheumatic diseases in Hong Kong, China. Arthritis \& Rheumatism 2011;63:1182-9.

[3] Souza DC, Santo AH, Sato El. Mortality profile related to systemic lupus erythematosus: a multiple cause-ofdeath analysis. Journal of Rheumatology 2012;39:496-503.

[4] Navarra SV, Leynes MS. Infections in systemic lupus erythematosus. Lupus 2010;19:1419-24. 386 S. Sciascia et al. / Best Practice \& Research Clinical Rheumatology 27 (2013) 377-389

[5] Zonana-Nacach A, Camargo-Coronel A, Yañez P, Sánchez L, Jimenez-Balderas FJ, Fraga A. Infections in outpatients with systemic lupus erythematosus: a prospective study. Lupus 2001;10:505-10.

*[6] Li Z, Chen L, Tao R, Fan X. Clinical and bacteriologic study of eighty-six patients with systemic lupus erythematosus complicated by infections. Chinese Medical Journal (England) 1998;111:913-6.

[7] Kang I, Park SH. Infectious complications in SLE after immunosuppressive therapies. Current Opinion in Rheumatology 2003;15:528-34.

*[8] Gladman DD, Hussain F, Ibañez D, Urowitz MB. The nature and outcome of infection in systemic lupus erythematosus. Lupus 2002;11:234-9.

[9] Pryor BD, Bologna SG, Kahl LE. Risk factors for serious infection during treatment with cyclophosphamide and high-dose corticosteroids for systemic lupus erythematosus. Arthritis \& Rheumatism 1996;39:1475-82.

[10] Nöel V, Lortholary O, Casassus P, Cohen P, Généreau T, André MH, et al. Risk factors and prognostic influence of infection in a single cohort of 87 adults with systemic lupus erythematosus. Annals of the Rheumatic Diseases 2001;60:1141-4.

[11] Bosch X, Guilabert A, Pallares L, Cervera R, Ramos-Casals M, Bové A, et al. Infections in systemic lupus erythematosus: a prospective and controlled study of 110 patients. Lupus 2006;15:584-9.

*[12] Barber C, Gold WL, Fortin PR. Infections in the lupus patient: perspectives on prevention. Current Opinion in Rheumatology 2011;23:358-65.

*[13] Ruiz-Irastorza G, Olivares N, Ruiz-Arruza I, Martinez-Berriotxoa A, Egurbide MV, Aguirre C. Predictors of major infections in systemic lupus erythematosus. Arthritis Research \& Therapy 2009;11:R109.

[14] Cervera R, Khamashta MA, Font J, Sebastiani GD, Gil A, Lavilla P, et al. Morbidity and mortality in systemic lupus erythematosus during a 5 -year period. A multicenter prospective study of 1,000 patients. European Working Party on Systemic Lupus Erythematosus. Medicine (Baltimore) 1999;78:167-75.

[15] Cervera R, Khamashta MA, Font J, Sebastiani GD, Gil A, Lavilla P, et al. European Working Party on Systemic Lupus Erythematosus. Morbidity and mortality in systemic lupus erythematosus during a 10-year period: a comparison of early and late manifestations in a cohort of 1,000 patients. Medicine (Baltimore) 2003 Sep;82(5):299-308.

[16] Edwards CJ, Lian TY, Badsha H, Teh CL, Arden N, Chng HH. Hospitalization of individuals with systemic lupus erythematosus: characteristics and predictors of outcome. Lupus 2003;12:672-6.

*[17] Naveau C, Houssiau FA. Pneumococcal sepsis in patients with systemic lupus erythematosus. Lupus 2005;14:903-6.

[18] Zandman-Goddard G, Shoenfeld Y. SLE and infections. Clinical Reviews in Allergy \& Immunology 2003 Aug;25:29-40.

[19] Petri M. Infection in systemic lupus erythematosus. Rheumatic Disease Clinics of North America 1998;24:423-56. 
[20] Goldblatt F, Yuste J, Isenberg DA, Rahman A, Brown J. Impaired C3b/iC3b deposition on Streptococcus pneumoniae in serum from patients with systemic lupus erythematosus. Rheumatology (Oxford) 2009;48:1498501.

[21] Tsao CH, Chen CY, Ou LS, Huang JL. Risk factors of mortality for salmonella infection in systemic lupus erythematosus. Journal of Rheumatology 2002;29:1214-8.

[22] Lim E, Koh WH, Loh SF, Lam MS, Howe HS. Non-thyphoidal salmonellosis in patients with systemic lupus erythematosus. A study of fifty patients and a review of the literature. Lupus 2001;10:87-92.

[23] Wu KC, Yao TC, Yeh KW, Huang JL. Osteomyelitis in patients with systemic lupus erythematosus. Journal of Rheumatology 2004;31:1340-3.

[24] Gerona JG, Navarra SV. Salmonella infections in patients with systemic lupus erythematosus: a case series. International Journal of Rheumatic Diseases 2009;12:319-23.

[25] Yinnon AM, Butnaru A, Raveh D, Jerassy Z, Rudensky B. Klebsiella bacteraemia: community versus nosocomial infection. Quarterly Journal of Medicine 1996;89:933-41.

[26] Chen MJ, Tseng HM, Huang YL, Hsu WN, Yeh KW, Wu TL, et al. Long-term outcome and short-term survival of patients with systemic lupus erythematosus after bacteraemia episodes: 6-yr follow-up. Rheumatology (Oxford) 2008;47:1352-7.

[27] George J, Shoenfeld Y. Infections, idiotypes and SLE. Lupus 1995;4:333-5.

[28] Isenberg DA, Maddison P, Swana G, Skinner RP, Swana M, Jones M, et al. Profile of autoantibodies in the serum of patients with tuberculosis, klebsiella and other gram-negative infections. Clinical \& Experimental Immunology 1987;67:516-23.

[29] Yun JE, Lee SW, Kim TH, Jun JB, Jung S, Bae SC, et al. The incidence and clinical characteristics of Mycobacterium tuberculosis infection among systemic lupus erythematosus and rheumatoid arthritis patients in Korea. Clinical and Experimental Rheumatology 2002;20:127-32.

[30] Hernández-Cruz B, Sifuentes-Osornio J, Ponce-de-León Rosales S, Ponce-de-León Garduño A, Díaz-Jouanen

E. Mycobacterium tuberculosis infection in patients with systemic rheumatic diseases. A case-series. Clinical and Experimental Rheumatology 1999;17:289-96.

[31] Prabu V, Agrawal S. Systemic lupus erythematosus and tuberculosis: a review of complex interactions of complicated diseases. Journal of Postgraduate Medicine 2010;56:244-50.

[32] Mok MY, Lo Y, Chan TM, Wong WS, Lau CS. Tuberculosis in systemic lupus erythematosus in an endemic area and the role of isoniazid prophylaxis during corticosteroid therapy. Journal of Rheumatology 2005;32:60915.

[33] Hou CL, Tsai YC, Chen LC, Huang JL. Tuberculosis infection in patients with systemic lupus erythematosus: pulmonary and extra-pulmonary infection compared. Clinical Rheumatology 2008;27:557-63.

[34] Sayarlioglu M, Inanc M, Kamali S, Cefle A, Karaman O, Gul A, et al. Tuberculosis in Turkish patients with systemic lupus erythematosus: increased frequency of extrapulmonary localization. Lupus 2004;13:274-8.

[35] Daher EF, Lima RS, Silva Júnior GB, Silva EC, Karbage NN, Kataoka RS, et al. Clinical presentation of leptospirosis: a retrospective study of 201 patients in a metropolitan city of Brazil. Brazilian Journal of Infectious Diseases Jan./Feb. 2010; 14(1). Salvador.

[36] Sieving RR, Kauffman CA, Watanakunakorn C. Deep fungal infection in system lupus erythematosus - three cases reported, literature reviewed. Journal of Rheumatology 1975;2:61-72.

[37] Cuchacovich R, Gedalia A. Pathophysiology and clinical spectrum of infections in systemic lupus erythematosus. Rheumatic Disease Clinics of North America 2009;35:75-93.

[38] Cheng HM, Huang DF, Leu HB. Disseminated nocardiosis with initial manifestation mimicking disease flareup of systemic lupus erythematosus in an SLE patient. American Journal of Medicine 2005;118:1297-8. S.

Sciascia et al. / Best Practice \& Research Clinical Rheumatology 27 (2013) 377-389 387

[39] Justiniano M, Glorioso S, Dold S, Espinoza LR. Nocardia brain abscesses in a male patient with SLE: successful outcome despite delay in diagnosis. Clinical Rheumatology 2007;26:1020-2. 
*[40] Ramos-Casals M, Cuadrado MJ, Alba P, Sanna G, Brito-Zerón P, Bertolaccini L, et al. Acute viral infections in patients with systemic lupus erythematosus: description of 23 cases and review of the literature. Medicine (Baltimore) 2008;87:311-8.

[41] Hsu TC, Tsay GJ. Human parvovirus B19 infection in patients with systemic lupus erythematosus. Rheumatology (Oxford) 2001;40:152-7.

[42] Sekigawa I, Nawata M, Seta N, Yamada M, lida N, Hashimoto H. Cytomegalovirus infection in patients with systemic lupus erythematosus. Clinical and Experimental Rheumatology 2002;20:559-64.

[43] Shahnaz S, Choksi MT, Tan IJ. Bilateral cytomegalovirus retinitis in a patient with systemic lupus erythematosus and endstage renal disease. Mayo Clinic Proceedings 2003;78:1412-5.

[44] Zandman-Goddard G, Shoenfeld Y. HIV and autoimmunity. Autoimmunity Reviews 2002;1:329-37.

[45] Palacios R, Santos J, Valdivielso P, Márquez M. Human immunodeficiency virus infection and systemic lupus erythematosus. An unusual case and a review of the literature. Lupus 2002;11:60-3.

[46] Carugati M, Franzetti M, Torre A, Giorgi R, Genderini A, Strambio de Castilla F, et al. Systemic lupus erythematosus and HIV infection: a whimsical relationship. Reports of two cases and review of the literature. Clinical Rheumatology 2013 Sep;32(9):1399-405.

[47] Sciascia S, Ceberio L, Garcia-Fernandez C, Roccatello D, Karim Y, Cuadrado MJ. Systemic lupus erythematosus and infections: clinical importance of conventional and upcoming biomarkers. Autoimmunity Reviews 2012;12:157-63.

[48] Firooz N, Albert DA, Wallace DJ, Ishimori M, Berel D, Weisman MH. High-sensitivity C-reactive protein and erythrocyte sedimentation rate in systemic lupus erythematosus. Lupus 2011;20:588-97.

*[49] O'Neill SG, Isenberg DA. Immunizing patients with systemic lupus erythematosus: a review of effectiveness and safety. Lupus 2006;15:778-83.

[50] van Assen S, Agmon-Levin N, Elkayam O, Cervera R, Doran MF, Dougados M, et al. EULAR recommendations for vaccination in adult patients with autoimmune inflammatory rheumatic diseases. Annals of the Rheumatic Diseases 2011;70: 414-22.

*[51] Abu-Shakra M. Safety of vaccination of patients with systemic lupus erythematosus. Lupus 2009;18:12058.

[52] Vista ES, Crowe SR, Thompson LF, Air GM, Robertson JM, Guthridge JM, et al. Influenza vaccination can induce new-onset anticardiolipins but not b2-glycoprotein-I antibodies among patients with systemic lupus erythematosus. Lupus 2012;21: 168-74.

[53] Crowe SR, Merrill JT, Vista ES, Dedeke AB, Thompson DM, Stewart S, et al. Influenza vaccination responses in human systemic lupus erythematosus: impact of clinical and demographic features. Arthritis \& Rheumatism 2011;63:2396-406.

[54] Holvast A, Huckriede A, Wilschut J, Horst G, De Vries JJ, Benne CA, et al. Safety and efficacy of influenza vaccination in systemic lupus erythematosus patients with quiescent disease. Annals of the Rheumatic Diseases 2006;65:913-8.

[55] Abu-Shakra M, Press J, Varsano N, Levy V, Mendelson E, Sukenik S, et al. Specific antibody response after influenza immunization in systemic lupus erythematosus. Journal of Rheumatology 2002;29:2555-7.

[56] Elkayam O, Paran D, Caspi D, Litinsky I, Yaron M, Charboneau D, et al. Immunogenicity and safety of pneumococcal vaccination in patients with rheumatoid arthritis or systemic lupus erythematosus. Clinical Infectious Diseases 2002;34: 147-53.

[57] Gaitonde S, Pathan E, Sule A, Mittal G, Joshi VR. Efficacy of isoniazid prophylaxis in patients with systemic lupus erythematosus receiving long term steroid treatment. Annals of the Rheumatic Diseases 2002;61:251-3. [58] Wiesik-Szewczyk E, Romanowska M, Mielnik P, Chwalinska-Sadowska H, Brydak LB, Olesi nska M, et al. Antiin fluenza vaccination in systemic lupus erythematosus patients: an analysis of specific humoral response and vaccination safety. Clinical Rheumatology 2010;29:605-13.

[59] Mathian A, Devilliers H, Krivine A, Costedoat-Chalumeau N, Haroche J, Huong DB, et al. Factors influencing the efficacy of two injections of a pandemic 2009 influenza $A$ (H1N1) nonadjuvanted vaccine in systemic lupus erythematosus. Arthritis \& Rheumatism 2011;63:3502-11. 
[60] Borba EF, Saad CG, Pasoto SG, Calich AL, Aikawa NE, Ribeiro AC, et al. Influenza A/H1N1 vaccination of patients with SLE: can antimalarial drugs restore diminished response under immunosuppressive therapy? Rheumatology (Oxford) 2012; 51:1061-9.

[61] Gatto M, Agmon-Levin N, Soriano A, Manna R, Maoz-Segal R, Kivity S, et al. Human papillomavirus vaccine and systemic lupus erythematosus. Clinical Rheumatology 2013 Sep;32(9):1301-7.

[62] Mok CC, Ho LY, Fong LS, To CH. Immunogenicity and safety of a quadrivalent human papillomavirus vaccine in patients with systemic lupus erythematosus: a case-control study. Annals of the Rheumatic Diseases 2013;72:659-64.

[63] Slade BA, Leidel L, Vellozzi C, Woo EJ, Hua W, Sutherland A, et al. Postlicensure safety surveillance for quadrivalent human papillomavirus recombinant vaccine. Journal of the American Medical Association 2009;302:750-7.

[64] Harpaz R, Ortega-Sanchez IR, Seward JF. Advisory Committee on Immunization Practices (ACIP) Centers for Disease Control and Prevention (CDC). Prevention of herpes zoster: recommendations of the Advisory Committee on Immunization Practices (ACIP). MMWR Recommendations and Reports 2008;57(RR-5):1-30. [65] Levin MJ, Gershon AA, Weinberg A, Song LY, Fentin T, Nowak B., Pediatric AIDS Clinical Trials Group 265 Team. Administration of live varicella vaccine to HIV-infected children with current or past significant depression of CD4(b) T cells. Journal of Infectious Diseases 2006;194:247-55.

[66] Weinberg A, Levin MJ, Macgregor RR. Safety and immunogenicity of a live attenuated varicella vaccine in VZVseropositive HIV-infected adults. Human Vaccines 2010;6:318-21.

[67] King SM, Saunders EF, Petric M, Gold R. Response to measles, mumps and rubella vaccine in paediatric bone marrow transplant recipients. Bone Marrow Transplant 1996;17:633-6.

*[68] Gilliland WR, Tsokos GC. Prophylactic use of antibiotics and immunisations in patients with SLE. Annals of the Rheumatic Diseases 2002;61:191-2.

[69] Zysset MK, Montgomery MT, Redding SW, Dell Italia L. Systemic lupus erythematosus: a consideration for antimicrobial prophylaxis. Oral Surgery, Oral Medicine, Oral Pathology 1987;64:30-4. 388 S. Sciascia et al. / Best Practice \& Research Clinical Rheumatology 27 (2013) 377-389

[70] Yoon KH, Fong KY, Tambyah PA. Fatal cytomegalovirus infection in two patients with systemic lupus erythematosus undergoing intensive immunosuppressive therapy: role for cytomegalovirus vigilance and prophylaxis? Journal of Clinical Rheumatology 2002;8:217-22.

[71] Gupta D, Zachariah A, Roppelt H, Patel AM, Gruber BL. Prophylactic antibiotic usage for Pneumocystis jiroveci pneumonia in patients with systemic lupus erythematosus on cyclophosphamide: a survey of US rheumatologists and the review of literature. Journal of Clinical Rheumatology 2008;14:267-72.

[72] Lertnawapan R, Totemchokchyakarn K, Nantiruj K, Janwityanujit S. Risk factors of Pneumocystis jiroveci pneumonia in patients with systemic lupus erythematosus. Rheumatology International 2009;29:491-6.

[73] Frank MM, Hamburger MI, Lawley TJ, Kimberley RP, Plotz PH. Defective reticuloendothelial system Fcreceptor dysfunction in systemic lupus erythematosus. New England Journal of Medicine 1979;300:518-23.

[74] Hepburn AL, Davies KA. Infection and SLE. Annals of the Rheumatic Diseases 2002;61:668-9.

[75] Bowness P, Davies KA, Norsworthy PJ, Athanassiou P, Taylor-Wiedeman J, Borysiewicz LK, et al. Hereditary C1q deficiency and systemic lupus erythematosus. Quarterly Journal of Medicine 1994;87:455-64.

*[76] Yong PF, Aslam L, Karim MY, Khamashta MA. Management of hypogammaglobulinaemia occurring in patients with systemic lupus erythematosus. Rheumatology (Oxford) 2008 Sep;47(9):1400-5.

[77] Venhoff N, Effelsberg NM, Salzer U, Warnatz K, Peter HH, Lebrecht D, et al. Impact of rituximab on immunoglobulin concentrations and B cell numbers after cyclophosphamide treatment in patients with ANCAassociated vasculitides. PLoS One 2012;7:e37626. 
Table 1.

Risk factors for infection.

Leukopenia

Acquired hypocomplementaemia

Genetic complement deficiency

$M B L$ deficiency

Hypogammaglobulinaemia

Splenectomy

Functional hyposplenism

Prednisolone dose

Immunosuppressive medication

Biologics e.g., rituximab

$\mathrm{MBL}=$ mannose binding lectin. 